\title{
Examination of directed flow as a signature of the softest point of the equation of state in QCD matter
}

\author{
Yasushi Nara, ${ }^{1,2}$ Harri Niemi, ${ }^{3}$ Akira Ohnishi, ${ }^{4}$ and Horst Stöcker ${ }^{2,3,5}$ \\ ${ }^{1}$ Akita International University, Yuwa, Akita-city 010-1292, Japan \\ ${ }^{2}$ Frankfurt Institute for Advanced Studies, D-60438 Frankfurt am Main, Germany \\ ${ }^{3}$ Institut für Theoretishe Physik, Johann Wolfgang Goethe Universität, D-60438 Frankfurt am Main, Germany \\ ${ }^{4}$ Yukawa Institute for Theoretical Physics, Kyoto University, Kyoto 606-8502, Japan \\ ${ }^{5}$ GSI Helmholtzzentrum für Schwerionenforschung GmbH, D-64291 Darmstadt, Germany
}

(Dated: September 27, 2016)

\begin{abstract}
We analyze the directed flow of protons and pions in high-energy heavy-ion collisions in the incident energy range from $\sqrt{s_{N N}}=7.7$ to $27 \mathrm{GeV}$ within a microscopic transport model. Standard hadronic transport approaches do not describe the collapse of directed flow below $\sqrt{s_{N N}} \simeq 20 \mathrm{GeV}$. By contrast, a model that simulates effects of a softening of the equation of state describes well the behavior of directed flow data recently obtained by the STAR Collaboration [1]. We give a detailed analysis of how directed flow is generated. Particularly, we found that softening of the effective equation of state at the overlapping region of two nuclei, i.e. the reaction stages where the system reaches high baryon density state, is needed to explain the observed collapse of proton directed flow within a hadronic transport approach.
\end{abstract}

PACS numbers: 25.75.-q, 25.75.Ld, 25.75.Nq, 21.65.+f

\section{INTRODUCTION}

Detecting the QCD phase transition is of primary interest in current nuclear and particle physics. Experiments have shown that a new form of strongly interacting matter is created in high-energy nuclear collisions at the Relativistic Heavy Ion Collider (RHIC) top energy and the Large Hadron Collider (LHC) energies [2]. Theoretical arguments and experimental signals imply that this matter is a quark-gluon plasma (QGP) created well above a transition temperature at almost zero baryon chemical potential. The lattice QCD calculations have confirmed that the transition from hadronic matter to QGP is a crossover at zero baryon density [3].

The next challenge is to discover the first and/or secondorder phase transition of QCD matter. Several effective model and approximate calculations of QCD suggest the existence of the first-order phase boundary and the critical point at finite chemical potentials [4]. Various observables, such as collective flows, particle ratios, moment of the distributions of conserved charges, have been measured at various incident energies to find evidences of a phase transition and a critical point [7, 8]. Particularly, we shall focus on the observation of collapse of directed flow by the STAR Collaboration [1], which might be a signal of a first-order phase transition at high baryon density region between hadronic matter and quark gluon plasma [6].

The collective transverse flow [9, 10] has been utilized to explore the properties of hot and dense matter, since it reflects the properties of the equation of state (EoS) in the early stages of nuclear collisions [11, 12]. The existence of bounce off, one of the collective flows, in heavy-ion collisions was first suggested in hydrodynamics [9-13] and examined in experiments at Bevalac [14] at Lawrence Berkeley National Laboratory. Later, the presence of the softest point near the phase transition in the EoS [5, 6] was discussed as a signal for the first-order phase transition, where the softest point is a local minimum of the ratio of pressure to energy density $p / \epsilon$ as a function of energy density, leading to a small sound velocity defined by $v_{s}^{2}=d p / d \epsilon$. In Ref. [15], baryon density dependence of the ratio $p / \epsilon$ was investigated within a quasi-particle model. The softest point of the crossover EoS at vanishing chemical potential is not very pronounced, but it is predicted that the EoS with a first order phase transition exhibits a very pronounced softest point at large chemical potentials.

Considerations of heavy ion collisions at RHIC energy have raised the question whether the physics of the often speculated first-order phase transition or the "softest point" should better be studied at moderate energies [11, 18] in which the collapse of the directed flow at $\sqrt{s_{N N}}=7-9 \mathrm{GeV}$ was speculated. Presumably, the EoS of baryon rich matter is the softest at moderate energy densities of a few $\mathrm{GeV} / \mathrm{fm}^{3}$.

Particularly, the excitation function of the directed flow slope with respect to rapidity $d v_{1} / d y$ decreases at $E_{\text {inc }}>$ $2 A \mathrm{GeV}$ [26] and is predicted to exhibit a minimum at a certain collision energy in hydrodynamical calculations using an EoS with a first-order QCD phase transition [6, 15, 18], where the $v_{1}$ is defined as the first Fourier component in the azimuthal angle distribution with respect to the reaction plane, $v_{1}=\langle\cos \phi\rangle$. The negative slope of $v_{1}$, called the third flow [16, 17] or the anti-flow of the nucleon [18, 19] emerges as a consequence of a tilted ellipsoid with respect to the beam axis from which negative flow builds up if matter passes through the softening point. Thus the collapse of the directed flow slope to a negative value might signal the first-order phase transition from hadron phase to quark-gluon phase, and was recently observed in the beam energy scan (BES) program performed at RHIC [1, 20].

A negative slope of proton $v_{1}$ at midrapidity has been found in the microscopic transport models RQMD [21], UrQMD [22], and PHSD/HSD [23] in which sign change is purely geometrical and only happens at large impact parameters and sufficiently higher collision energies. Note that such microscopic transport models do not show a negative slope of the proton $v_{1}$ at midrapidity at bombarding energies of 
$\sqrt{s_{N N}} \leq 20 \mathrm{GeV}$ [23-25], and thus the negative slope of the proton $v_{1}$ at $\sqrt{s_{N N}}=11.5$ and $19.6 \mathrm{GeV}$ observed by the STAR Collaboration [1] is incompatible with the predictions by the standard hadronic transport models. We also note that the hadronic transport model with momentum dependent mean field significantly improves the description of the directed flow data from E895 [26] and NA49 data [27, 28], but inclusion of the hadronic mean field does not lead to the negative proton $d v_{1} / d y$ [29]. This gives indirect evidence of the phase transition around such collision energies.

The excitation function of the directed flow slope was investigated also in a transport + hydrodynamics hybrid approach [25], where it was found that there is no sensitivity of the directed flow on the EoS, and there is no minimum in the excitation function of the directed flow slope. In contrast, strong sensitivities of the directed flow to the EoS are found in a three-fluid model [30]. The three-fluid calculations indicate that the crossover deconfinement transition is consistent with the directed flow data of energy range up to $\sqrt{s_{N N}} \approx 11.5 \mathrm{GeV}$. However, the PHSD transport model which incorporates crossover EoS does not show the experimentally observed minimum [23]. Thus it is not yet clear whether the negative slope of $v_{1}$ signals the softening of the EoS in hybrid approaches.

In the present paper, we investigate the directed flow in the BES energy region within the microscopic transport model JAM [32] by imposing attractive orbits for each two-body scattering to simulate effects of a softening of the EoS.

\section{MODEL}

The hadronic transport model JAM [32] has been developed based on resonance and string production and their decay, which is similar to other transport models [33-35]. Secondary products from decay can interact with each other by binary collisions. A detailed description of the JAM model can be found in Ref. [32].

We take into account nuclear EoS effects within a microscopic transport approach by changing the standard stochastic two-body scattering style, which is normally implemented so as not to contribute to the pressure. For example, we can simulate repulsive $N N$ potential effects by allowing only repulsive orbits in the two-body collisions [36-38], instead of choosing the scattering angle randomly as in a standard cascade. It is reported that directed flow at Bevalac and Alternating Gradient Synchrotron (AGS) energies are well described by this approach [38]. Later, attractive orbits were introduced to effectively incorporate the softening of the EoS [39] guided by the virial theorem [40]. In this way, different treatment of scatterings can modify the EoS.

We impose attractive orbits for each two-body hadronhadron scattering to reduce the pressure of the system. The pressure of a system, in which particles are interacting with each other only by two-body scattering, is given by the virial theorem as [40]

$$
P=P_{f}+\frac{1}{3 T V} \sum_{(i, j)}\left(\boldsymbol{q}_{i} \cdot \boldsymbol{r}_{i}+\boldsymbol{q}_{j} \cdot \boldsymbol{r}_{j}\right)
$$

where $P_{f}=1 /(3 V T) \int d t \sum_{i} \boldsymbol{p}_{i} \cdot \boldsymbol{v}_{i}$ corresponds to the free streaming contribution. The second term represents the pressure generation from all two-body scatterings between the pair of particles $i$ and $j$, where $\boldsymbol{q}_{i}=-\boldsymbol{q}_{j}=\boldsymbol{p}_{i}^{\prime}-\boldsymbol{p}_{i}$ is the momentum transfer and $\boldsymbol{r}_{i}$ and $\boldsymbol{r}_{j}$ are the coordinate of colliding particles. $V$ is the volume of the system, and $T$ is a time interval over which the system is measured. Thus, pressure generation by the two-body collisions is related to the scattering style; the repulsive orbit $\boldsymbol{q}_{i} \cdot\left(\boldsymbol{r}_{i}-\boldsymbol{r}_{j}\right)>0$ enhances the pressure, while the attractive orbit $\boldsymbol{q}_{i} \cdot\left(\boldsymbol{r}_{i}-\boldsymbol{r}_{j}\right)<0$ reduces the pressure. Note that an attractive potential softens the EoS [31], which leads to an attractive orbit.

Attractive orbits are realized in the simulation as follows. Each orbit is selected randomly as in the standard simulation, but in the case where the orbit is repulsive, we change it to an attractive one by exchanging the momentum of two particles in the two-body cener-of-mass (c.m.) frame. Thus the scattering rate remains the same. While in reality modification of the scattering style should depend for example, on variables such as local energy density, we impose a modified scattering style for all hadron-hadron $2 \rightarrow 2$ scatterings in order to see an effect of the softening, instead of trying to fit the data. Thus there is no adjustable free parameter in our current approach unlike Ref. [38] in which repulsive trajectories are selected for colliding baryons with some probability in order to generate more pressure at AGS energies.

An energy density dependent implementation of attractive orbits will be examined shortly in Sec. IIIF

\section{RESULTS}

We now discuss directed flows in the BES energy region. In the simulation, we choose the impact parameter range $4.6<$ $b<9.4 \mathrm{fm}$ for mid-central and $0<b<4.0 \mathrm{fm}$ for central collisions for the STAR data [1].

\section{A. Beam energy dependence of directed flow}

In Fig. 11 we show the calculated directed flow $v_{1}$ of protons and pions in mid-central collisions from the standard JAM cascade (dotted lines) and the JAM cascade with attractive orbits (solid line) in Au+Au collisions at $\sqrt{s_{N N}}=$ 7.7, 11.5, 19.6 and $27 \mathrm{GeV}$ in comparison with the data from STAR Collaboration [1]. The standard JAM cascade calculation agrees with the $7.7 \mathrm{GeV}$ data. However, it is seen that $v_{1}$ from the standard JAM cascade calculations for beam energies of 11.5 and $19.6 \mathrm{GeV}$ yields much larger $v_{1}$ than the STAR data. The proton slope from JAM turns out to be negative at $\sqrt{s_{N N}}=27 \mathrm{GeV}$. This is because of the geometrical reason pointed out in Ref. [21], and is not related to the softening of the EoS within our transport approach. We note that 


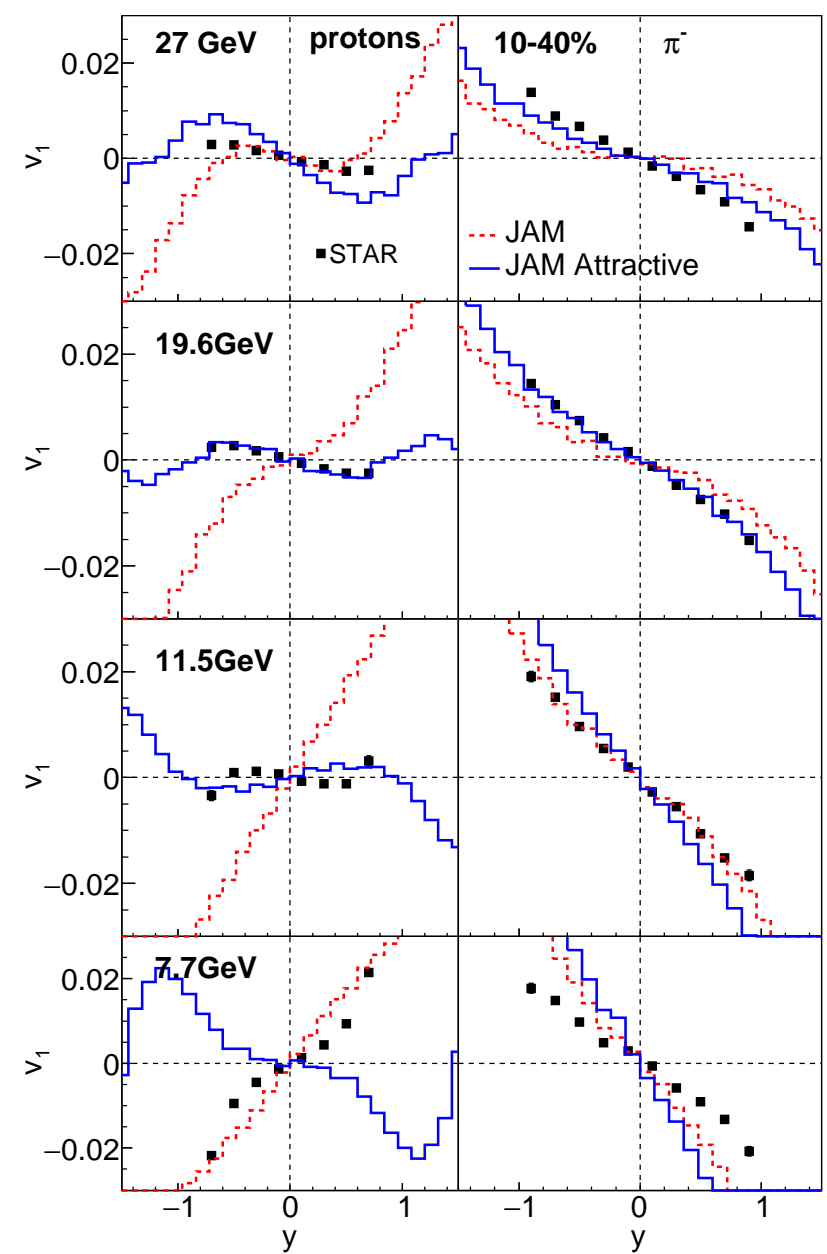

FIG. 1: Directed flows of protons and pions in mid-central $\mathrm{Au}+\mathrm{Au}$ collisions $(10-40 \%)$ at $\sqrt{s_{N N}}=7.7-27 \mathrm{GeV}$ from the JAM cascade mode (dashed lines) and the JAM cascade with attractive orbits (solid lines) in comparison with the STAR data [1].

our results are consistent with other microscopic transport approaches [23-25].

By comparison, attractive orbit scatterings drastically reduce the $v_{1}$ slope, and explain the STAR data at $\sqrt{s_{N N}} \gtrsim$ $10 \mathrm{GeV}$ as shown in Fig. 1 (solid lines); at $\sqrt{s_{N N}}=11.5$ and $19.6 \mathrm{GeV}$, the $v_{1}$ slope becomes almost zero and negative, respectively. At lower energy $\sqrt{s_{N N}}=7.7 \mathrm{GeV}$, results with attractive orbits are far from the data, and there should not be large EoS softening.

From this analysis, we find that the softening of the EoS affects the directed flow of protons at midrapidity and should emerge in the beam energy range of $\sqrt{s_{N N}} \gtrsim 10 \mathrm{GeV}$, but its effects should be small at $\sqrt{s_{N N}}=7.7 \mathrm{GeV}$. Since NA49 data at $\sqrt{s_{N N}}=8.9 \mathrm{GeV}$ may also indicate evidence of softening of the EoS [24], the onset beam energy of the softening might be lower than $10 \mathrm{GeV}$. Therefore, detailed experimental studies are needed around the beam energies of $\sqrt{s_{N N}} \lesssim 10$ GeV.

Unfortunately, the EoS softening effects are not easy to see when the $v_{1}$ slope is already negative in the standard cascade.
As seen in Fig. 11, the proton $v_{1}$ slope at $\sqrt{s_{N N}}=27 \mathrm{GeV}$ and pion $v_{1}$ slopes are negative in the standard cascade from geometrical non-QGP effects [21] and from absorption by baryons [41], respectively. It should be noted, however, that JAM with attractive orbits overestimates the negative slope of the proton $v_{1}$ indicating the need to reharden the EoS, i.e.; matter created at this collision energy reaches well above the transition region or weak softening of the EoS due to less net baryonic density.

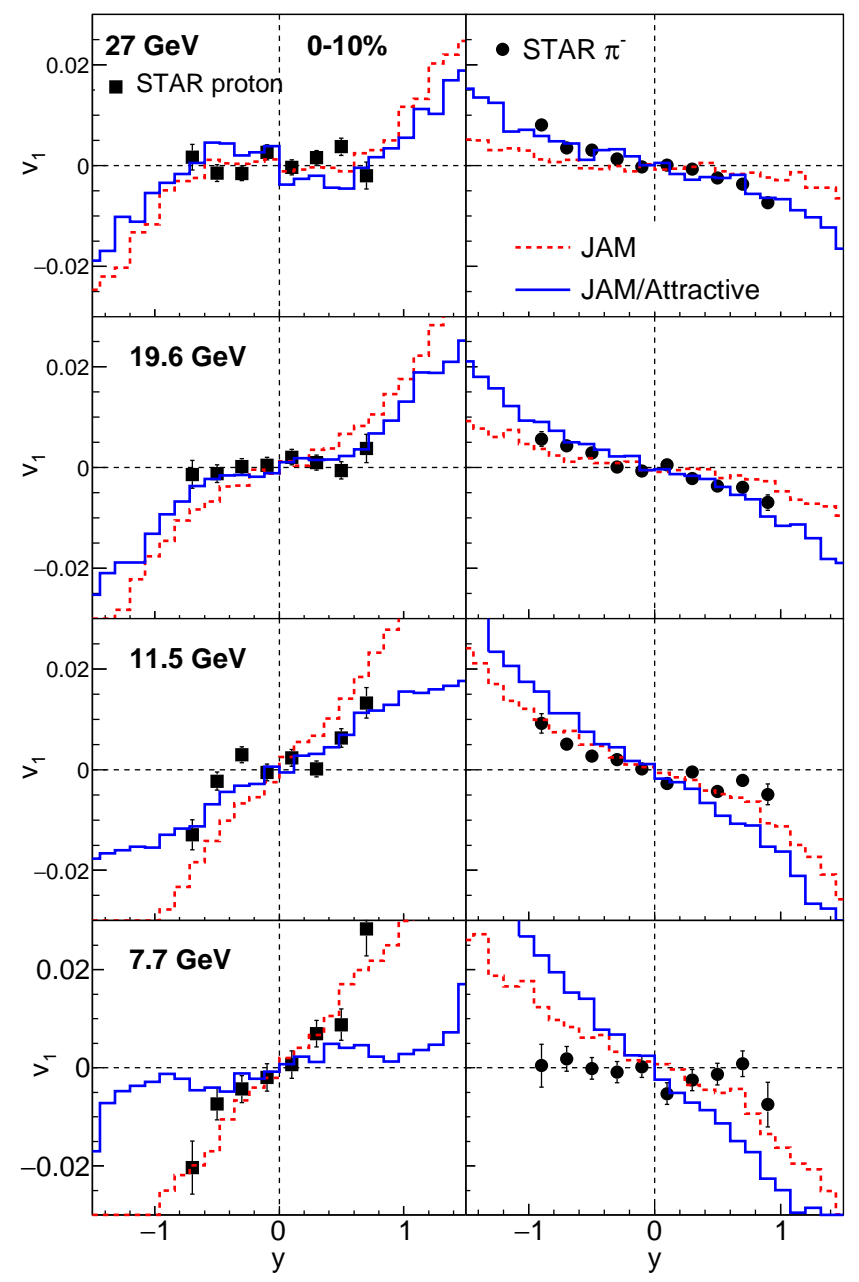

FIG. 2: Same as in Fig.1 but for central collisions (0-10\%).

We now discuss the directed flows in central collisions in order to distinguish the geometrical effects from phase transition. Since sign change of the proton $v_{1}$ is purely geometrical and only happens at large impact parameters in standard hadronic transport models, it is possible to find the effects of the softening sharply in central collisions. We show directed flows in central collisions in Fig. 2 STAR data on the proton $v_{1}$ do not show negative slope for central collisions. The standard JAM cascade describes well the data at $7.7 \mathrm{GeV}$, indicating that the hadronic description may be reasonable at 7.7 $\mathrm{GeV}$. It is seen that the proton $v_{1}$ at $27 \mathrm{GeV}$ from both JAM with attractive orbits and standard JAM simulation yield neg- 
ative slope. Thus 7.7 and $27 \mathrm{GeV}$ data do not show a hint of the softening of the EoS within our analysis.

On the other hand, one sees that JAM with attractive orbits again quite reasonably describes the data at 11.5 and 19.6 GeV, while the standard JAM cascade overestimates the data. Therefore, STAR data on the proton directed flow for both central and mid-central collisions indicate evidence of the softening of the EoS.

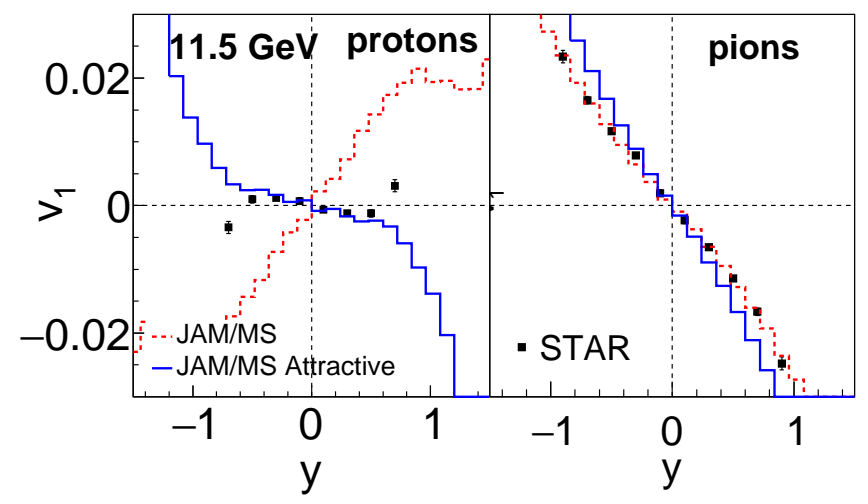

FIG. 3: Directed flows of protons (left) and pions (right) in midcentral $\mathrm{Au}+\mathrm{Au}$ collisions $(10-40 \%)$ at $\sqrt{s_{N N}}=11.5 \mathrm{GeV}$. In the JAM calculations, momentum dependent hadronic mean-field potentials are included (JAM/MS). The dotted lines correspond to the result from the standard JAM/MS model, while the solid lines are for JAM/MS with attractive orbit results. Symbols show STAR data [1].

It is also necessary to examine the influence of the nuclear mean field on the directed flow at midrapidity, since the mean field can also modify the flows. Nuclear mean fields of hadrons are included based on the framework of simplified version of relativistic quantum molecular dynamics (RQMD/S) in Ref. [29]. Density dependent Skyrme-type and momentum dependent Yukawa potentials are employed as in Ref. [29], but with slightly different parameter sets which yields the incompressibility of $K=272 \mathrm{MeV}$ [42].

In Fig. 3, we show the calculated results of the directed flow of protons and pions at $\sqrt{s_{N N}}=11.5 \mathrm{GeV}$ from JAM with momentum dependent potentials (JAM/MS) together with the STAR data. The mean field slightly reduces the proton directed flow, but the basic trend is the same as the JAM cascade result. It is interesting to see that attractive orbits supplemented by the mean field yields negative slope, and provides a better description of the data than the cascade calculation at midrapidity.

\section{B. Effective EoS}

We would like to see how much pressure is suppressed by imposing attractive orbits. The free streaming part of the local isotropic pressure $P_{f}$ can be computed from the the energymomentum tensor $T^{\mu \nu}=\sum_{h} \int \frac{d^{3} p}{p^{0}} p^{\mu} p^{\nu} f_{h}(x, p)$ as $P_{f}=$ $-\frac{1}{3} \Delta_{\mu \nu} T^{\mu \nu}$, with the projector of $\Delta^{\mu \nu}=g^{\mu \nu}-u^{\mu} u^{\nu}$, where $u^{\nu}$ is a hydrodynamics velocity defined by the Landau and

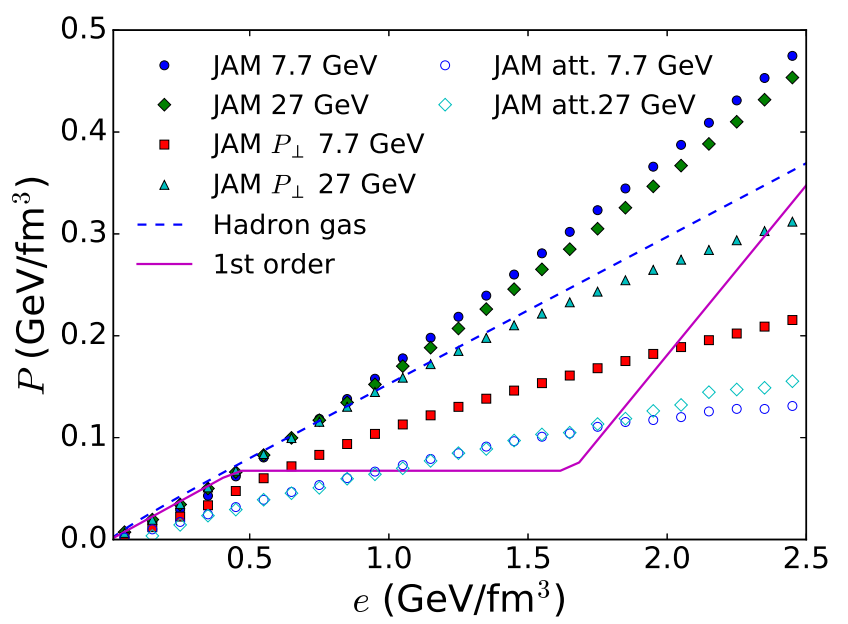

FIG. 4: Effective EoS extracted from the time evolution of simulations in $\mathrm{Au}+\mathrm{Au}$ collisions at $\sqrt{s_{N N}}=7.7$ and $27 \mathrm{GeV}$. Full (open) circles and full (open) diamonds represent the pressures $P$ at from standard JAM (JAM with attractive orbits) at 7.7 and $27 \mathrm{GeV}$, respectively. Triangles and boxes represent the transverse part of the pressure $P_{\perp}$ for the JAM standard at 7.7 and $27 \mathrm{GeV}$, respectively. The dashed and sold lines represent the EoS from hadron gas and the EoS with a first-order phase transition used in Ref. [44].

Lifshitz definition that may be solved iteratively [43]. The pressure difference $\Delta P$ from the free streaming $P_{f}$ caused by the two-body collision between particles $i$ and $j$ at the spacetime coordinates of $q_{i}$ and $q_{j}$ is estimated based on the formula given by Ref. [39]:

$$
\Delta P=-\frac{\rho}{3\left(\delta \tau_{i}+\delta \tau_{j}\right)}\left(p_{i}^{\prime}-p_{i}\right)^{\mu}\left(q_{i}-q_{j}\right)_{\mu},
$$

where $\rho=N^{\nu} u_{\nu}, N^{\nu}=\sum_{h} \int \frac{d^{3} p}{p^{0}} p^{\nu} f_{h}(x, p)$ is the Lorentz invariant local particle density, $\delta \tau_{i}$ is the proper time interval between successive collisions, and $p_{i}^{\prime}-p_{i}$ is the energymomentum change of the particle $i$. We extract the "effective EoS" by accumulating statistics by computing local pressure $P=P_{f}+\Delta P$ and energy density $e=u_{\mu} T^{\mu \nu} u_{\nu}$ at each collision point in the JAM simulation in the central region of the reaction zone specified by the longitudinal region $|z|<1 \mathrm{fm}$ and the transverse radius of less than $3 \mathrm{fm}$ in $\mathrm{Au}+\mathrm{Au}$ collisions at $\sqrt{s_{N N}}=7.7,11.5,19.6$, and $27 \mathrm{GeV}$. We have checked that the volume dependence on the EoS extracted from the simulation is very weak.

In Fig. 4 pressure $P$ is plotted as a function of energy density $e$ from JAM simulations as well as the ideal hadron gas EoS and the EoS with a first-order phase transition (EOSQ) [44] at vanishing baryon chemical potential. We first discuss the EoS in the standard JAM simulation. We see some beam energy dependence of the effective EoS as reported in Ref. [45] in which the EoS was extracted to be $P \simeq(0.12-0.15) e$ from AGS to Super Proton Synchrotoron (SPS) energies that is quite similar to our results. There is a deviation of the effective EoS from the hadron resonance gas EoS at higher energy densities, which is mainly due to the nonequilibrium evolution of the system since the high energy 
density parts are extracted from early times where the system is far from the equilibrium state. In particular, pressure in the compression stage of the reaction tends to be much higher than the values expected from the equilibrium EoS. We note that the transverse pressure $P_{\perp}=\left(T_{L R}^{x x}+T_{L R}^{y y}\right) / 2$, where $T_{L R}^{i i}$ is the energy-momentum tensor at a local rest frame, is lower than the equilibrium hadron resonance gas EoS in high energy density regions as seen in our results in Fig. 4 which are also reported in Ref. [46]. It is expected that the deviation of the effective pressure in JAM compared to the equilibrium hadron gas mainly comes from the bulk viscous pressure and/or chemical composition. Note that the difference between transverse and longitudinal pressure (shear-stress tensor) should not contribute to the isotropic pressure defined through the isotropic projection of the energy-momentum tensor. In the compression phase, the bulk pressure should give a positive correction to the equilibrium pressure, but in the expansion stage, the sign should change. Judging by Fig. 4, such sign change is, at least, not very pronounced. So it indicates that the main contribution to the hardening of the EoS at high energy densities in JAM is from the chemical composition; namely, the system at early stages of the reaction is highly out of the chemical equilibrium state.

At lower energy densities, transverse pressure $P_{\perp}$ is close to the isotropic pressure $P$, showing the kinetic equilibration of the system, and transverse pressure departs from isotropic pressure above $e \approx 0.3-1.0 \mathrm{GeV} / \mathrm{fm}^{3}$ depending on the beam energy. Chemical equilibrium of the system in the time evolution of hadron transport models was investigated in Ref. [45], and it was found that it reaches at late times, which is consistent with the finding here that the effective EoS from standard JAM is close to the ideal hadron resonance EoS at lower energy densities. After equilibration, pressure from standard JAM approaches values close to the ideal hadron resonance gas EoS which is seen in Fig.4 when the energy density is less than $1 \mathrm{GeV} / \mathrm{fm}^{3}$. As energy density drops further, the standard JAM yields slightly less pressure than that of the ideal gas EoS, because of the chemical freeze-out [46].

The effective EoS from JAM with attractive orbits is compared with the EoS from the standard JAM simulation in Fig. 4. When attractive orbits are selected for all two-body scatterings in JAM, we see a significant reduction of the pressure, yielding a similar amount of softening in the transition region as EOS-Q, although our effective EoS does not exhibit a sharp first-order phase transition, since we do not impose any specific condition to allow for an attractive orbit. We will examine which part of the EoS is responsible for the collapse of proton directed flow in the next sections.

\section{Generation of directed flow}

Let us now examine where the negative slope is generated. Time evolution of the sign weighted directed transverse momentum integrated over the rapidity range of $-1<y<1$,

$$
v_{1}^{*}=\int_{-1}^{1} d y v_{1}(y) \operatorname{sgn}(y)
$$

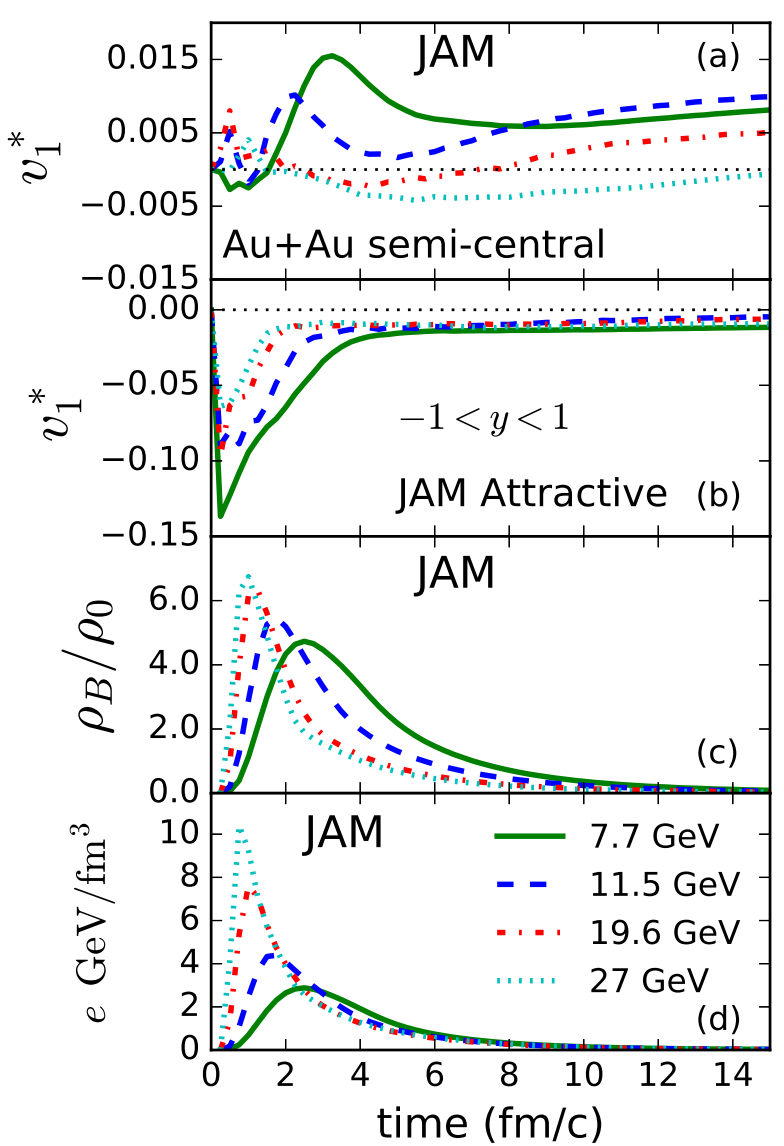

FIG. 5: Time evolution of sign weighted $v_{1}^{*}$ integrated over midrapidity for baryons in semicentral $\mathrm{Au}+\mathrm{Au}$ collisions for $\sqrt{s_{N N}}=$ 7.7, 11.5, 19.6, $27 \mathrm{GeV}$ from (a) standard JAM and (b) JAM with attractive orbits simulations. Time evolutions of normalized netbaryon density and energy density from the standard JAM calculations are shown in the panels (c) and (d), respectively. Baryon density and energy density are averaged over a cylindrical volume of transverse radius $3 \mathrm{fm}$ and longitudinal distance of $1 \mathrm{fm}$ centered at the origin. Those particles which have not interacted yet are not included in the calculations of $v_{1}^{*}, \rho_{B}$, and $e$.

for baryons is displayed in Fig. 5 in semicentral Au+Au collision for both the standard JAM cascade and JAM with attractive orbits. In the standard JAM cascade, directed flow of baryons rises in the early states of the reaction before two nuclei pass through each other, and decreases with time. At late times, it rises slowly again with time.

In the JAM cascade with attractive orbits, on the other hand, it is observed that directed flow is strongly modified to be negative by the reduction of pressure at early times, especially at lower energies, and directed flow always increases as a function of reaction time. We note that the slope of directed flow stays negative also in the rescattering stages of the reaction; the period long after two nuclei pass through each other. As expected, directed flow becomes smaller as collision energy becomes higher, due to less interaction time [17]. 


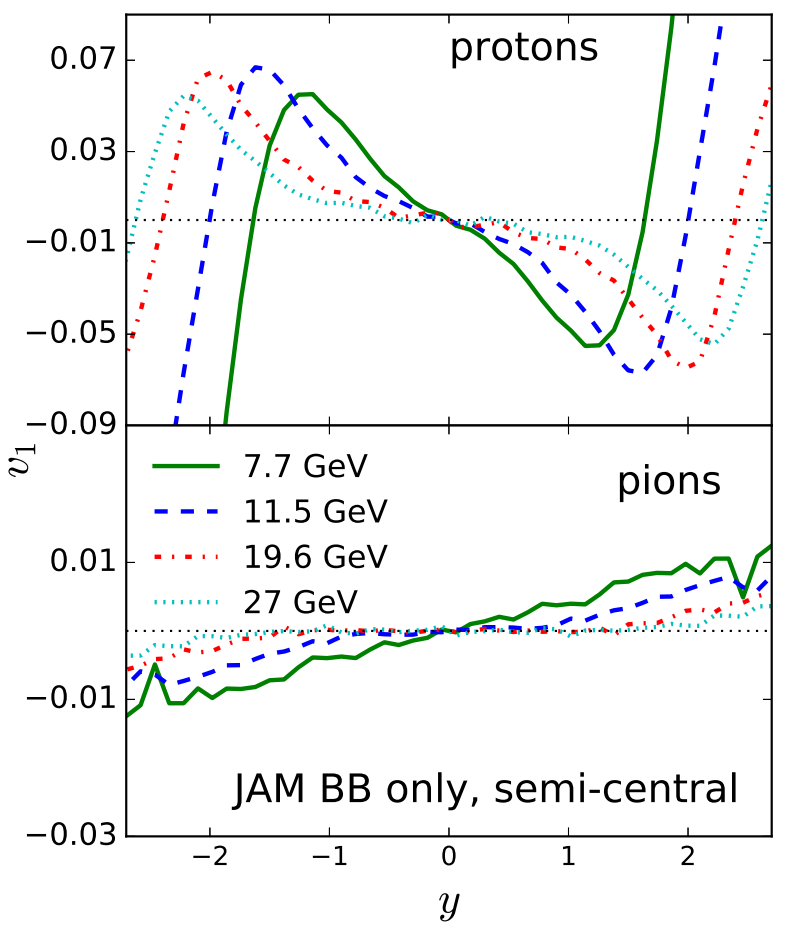

FIG. 6: Directed flows of protons and pions in midcentral $\mathrm{Au}+\mathrm{Au}$ collisions collisions (10-40\%). The lines correspond to the results from the JAM cascade model with only baryon-baryon collisions included.

\section{Effects of meson-baryon interactions}

The range of beam energies covered by BES is quite interesting. The crossing times of the two gold nuclei in the $\mathrm{c}$. $\mathrm{m}$. frame are approximately $3.27,2.15,1.25$, and $0.906 \mathrm{fm} / c$ at $\sqrt{s_{N N}}=7.7,11.5,19.6$ and $27 \mathrm{GeV}$, respectively. Thus, hadronic rescatterings start in $\mathrm{Au}+\mathrm{Au}$ collisions before passing through two nuclei at $\sqrt{s_{N N}}=7.7 \mathrm{GeV}$, because crossing time is much longer than the hadronization time from string fragmentation which is typically $1 \mathrm{fm} / c$. On the other hand, at $\sqrt{s_{N N}}=27 \mathrm{GeV}$, most of the hadronic rescattering among produced particles occur after two nuclei pass through each other. In Ref. [21], a wiggle structure in the rapidity dependence of proton directed flow was predicted at $\sqrt{s_{N N}}=200$ $\mathrm{GeV}$ in which initial nucleon-nucleon collisions are well isolated from the late hadronic rescatterings, and it is argued that a wiggle structure appears as a result of the correlation between the position of a nucleon and its stopping power due to initial Glauber type nucleon-nucleon collisions. The negative directed flow seen in the hadronic transport models at $\sqrt{s_{N N}}=27 \mathrm{GeV}$ is due to the same reason. At energies $\sqrt{s_{N N}} \leq 19.6 \mathrm{GeV}$, this correlation is contaminated by meson-baryon collisions, since mesons and baryons start interacting with each other before the two nuclei pass though each other.

We now look at the effects of rescatterings between mesons and baryons in the BES energy region. In Fig. 6, we show the rapidity dependence of $v_{1}$ for both protons and pions for

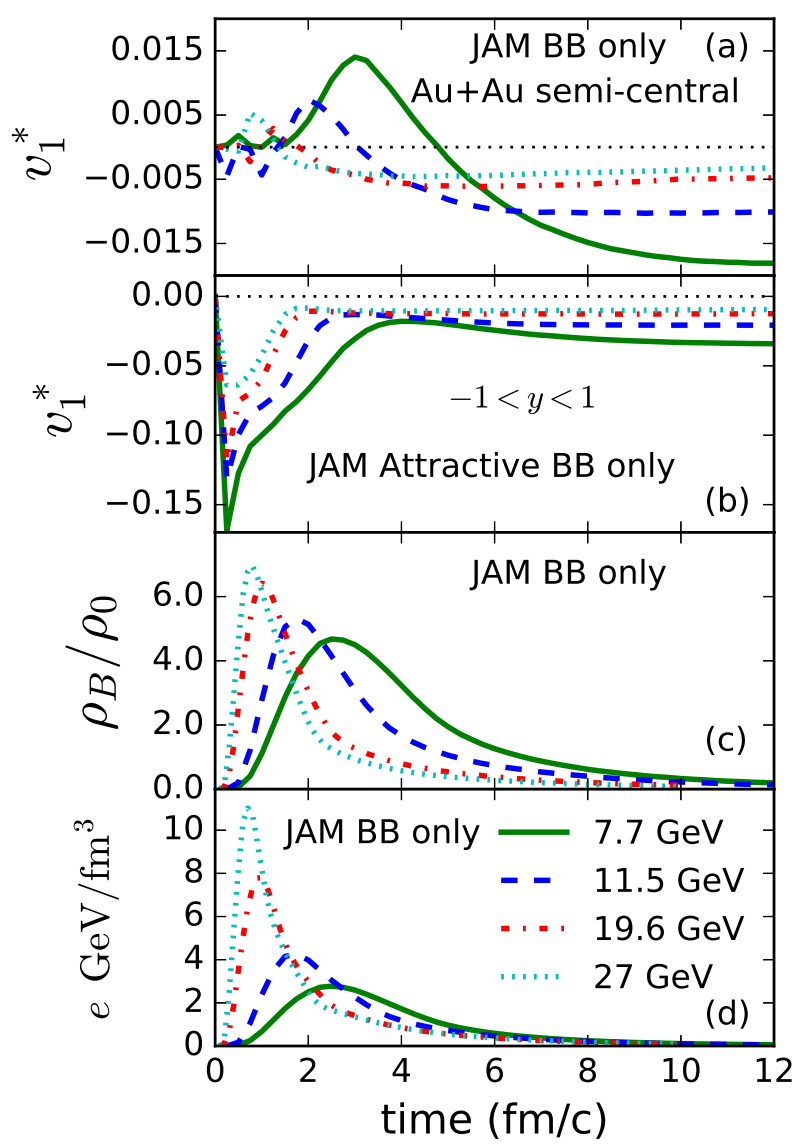

FIG. 7: Same as Fig. 5 but JAM cascade simulations with only baryon-baryon collisions.

the JAM simulation without meson-baryon, and meson-meson collisions. We see the wiggle structure in the rapidity dependence of the proton $v_{1}$ for all of the beam energies, and pion directed flow is very small but slightly positive. The appearance of wiggle structure at lower beam energies when one switches off meson-baryon scatterings may be partly due to the similar mechanism as pointed out by Ref. [21]. Thus we conclude that baryon-baryon collisions alone generates negative proton directed flow at midrapidity, and meson-baryon collisions bring the proton directed flow to the positive side and the pion directed flow to the negative side. This implies that strong negative directed flow must be generated at the initial stages of nuclear collisions to get the negative proton flow at freeze-out.

In order to examine further in detail how $v_{1}$ is generated, we plot in Fig. 7 the time evolution of $v_{1}^{*}(|y|<1)$ from the JAM simulations by switching off all baryon-meson and meson-meson collisions (JAM BB only). The upper panel of Fig. 7 shows the time evolutions of $v_{1}^{*}$ from JAM simulations with only baryon-baryon collisions. It shows that positive directed flow is first generated before two nuclei pass though each other, and then it becomes negative in the expansion stages of the reaction. The lower beam energy yields larger negative directed flow. This is because of the effect of 
spectators as well as the increasing number of baryon-baryon collisions at lower beam energies. The average number of baryon-baryon collisions $\left\langle N_{\text {coll }}\right\rangle$ in $\mathrm{Au}+\mathrm{Au}$ semicentral collisions is larger at lower beam energy: $\left\langle N_{\text {coll }}\right\rangle=490,400,340$, and 310 for $\sqrt{s_{N N}}=7.7,11.5,19.6$ and $27 \mathrm{GeV}$, respectively.

In the middle panel of Fig.7 we display the time evolution of $v_{1}^{*}$ in JAM BB only with attractive orbits. As in the case of full simulation, strong negative directed flow is generated in the early stages of the reaction and it rises in time, then it stays the same value at later times because of the absence of hadronic rescatterings. The question then arises, which part of the reaction stage is more relevant for the negative directed flow of protons?

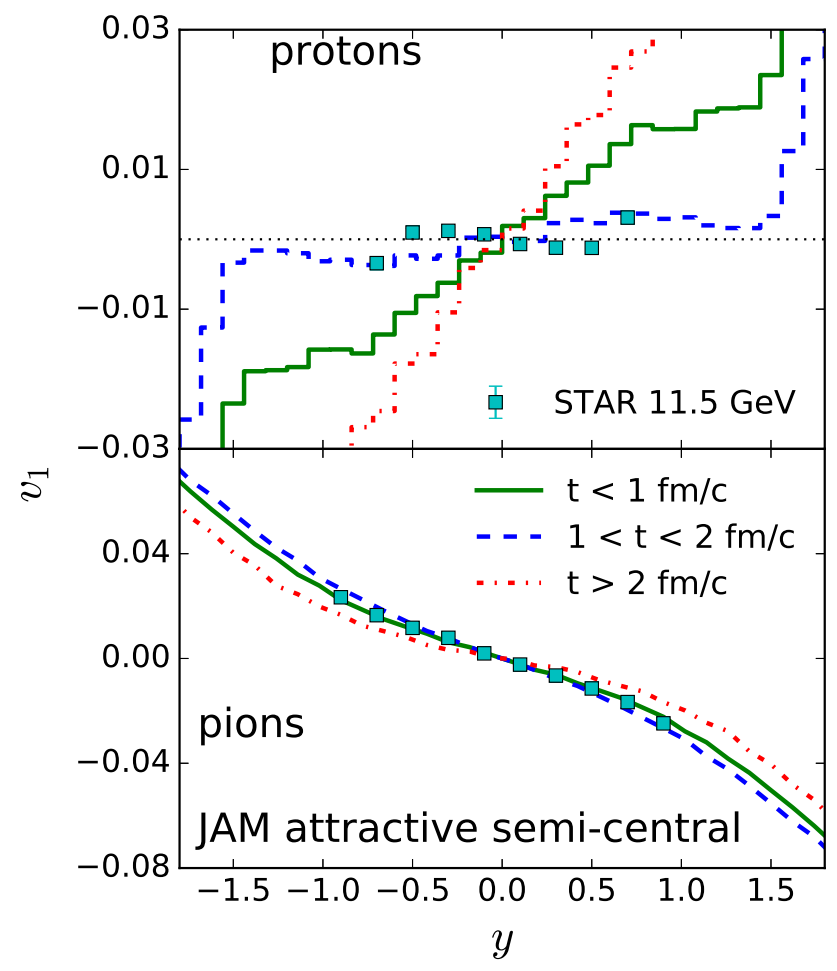

FIG. 8: Rapidity distribution of directed flow $v_{1}$ for protons and pions in semicentral $\mathrm{Au}+\mathrm{Au}$ collisions for $\sqrt{s_{N N}}=11.5 \mathrm{GeV}$ from JAM simulation in which attractive orbits are imposed at different time intervals; the solid line corresponds to the simulation with attractive orbits for time $t<1 \mathrm{fm} / c$, the dashed line for $1<t<2$ $\mathrm{fm} / c$, and the dotted line for $t>2 \mathrm{fm} / c$.

To try to answer the question, we plot in Fig. 8 the rapidity dependence of $v_{1}$ in $\mathrm{Au}+\mathrm{Au}$ semicentral collision for three different JAM simulations at $\sqrt{s_{N N}}=11.5 \mathrm{GeV}$ by noting that the crossing time of two nuclei is about $2.15 \mathrm{fm} / \mathrm{c}$ : (1) JAM with attractive orbits only for compression stages of the reaction until two nuclei reach full overlap, i.e. time less than $1 \mathrm{fm} / c$; (2) JAM with attractive orbits at the reaction time between 1 and $2 \mathrm{fm} / c$ which corresponds to the time range with largest baryon densities. (3) JAM with attractive orbits at times later than $2 \mathrm{fm} / c$; and As we expect, when attractive orbits are imposed at times later than $2 \mathrm{fm} / c$, it is too late to generate negative flow, and the result is almost identical to the standard JAM simulation. Furthermore, it is very interesting to see that the effect of attractive orbits at times earlier than $1 \mathrm{fm} / \mathrm{c}$ is small and its effect alone cannot explain the strong suppression of the flow. To see this point more clearly, time evolution of $v_{1}^{*}$ is displayed in Fig. 9. One see that strong negative directed flow generated in the earliest stages of the reaction quickly disappears with a much faster rate than the one shown in the lower panel of Fig. 5. Thus initial scatterings at the compression stage of the reaction are not important in generating negative $v_{1}$. Finally, it is shown that what is responsible for the strong suppression of the proton flow is the effect of attractive orbits in the time interval $1<t<2 \mathrm{fm} / c$, which coincides with the highest baryon density in the course of the reaction at $\sqrt{s_{N N}}=11.5 \mathrm{GeV}$. This effect can be further confirmed in the time evolution of $v_{1}^{*}$ in Fig. 9 Attractive orbits at this stage are important to suppress the rise of $v_{1}$ due to the hadronic rescatterings.
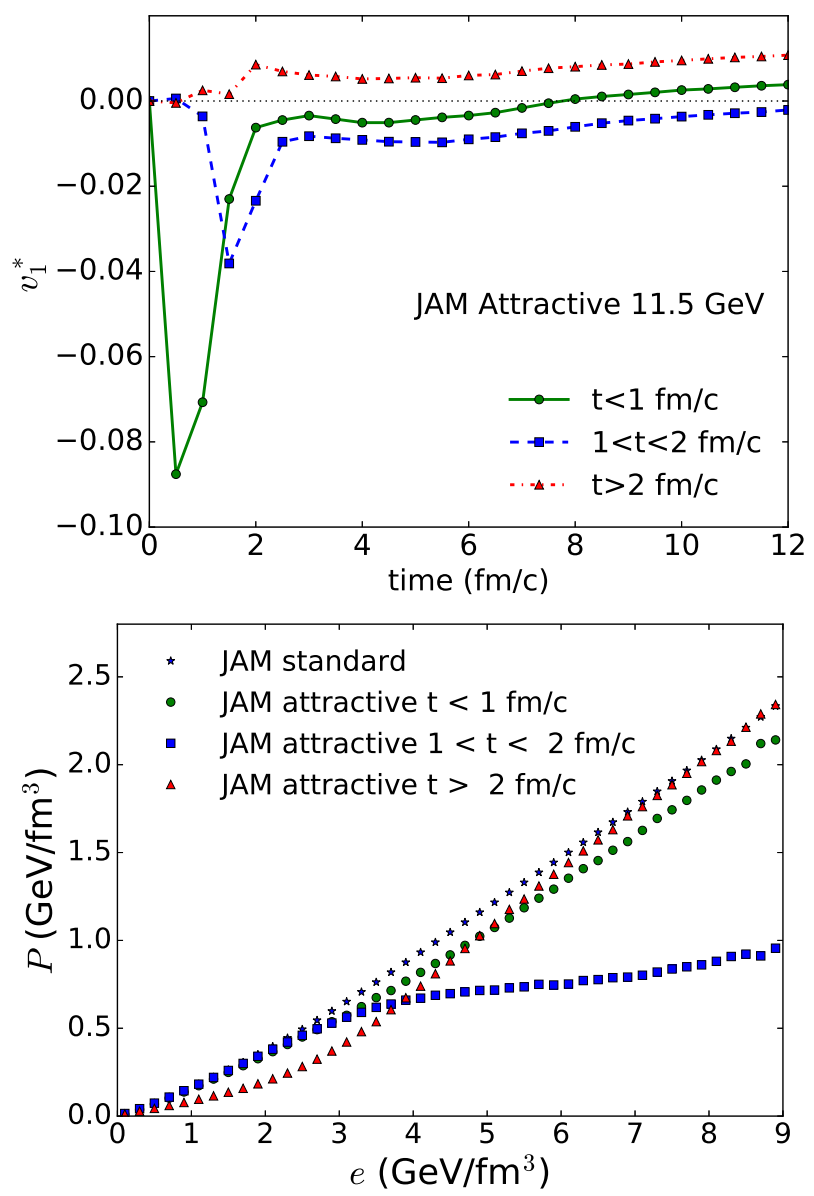

FIG. 9: Time evolution of sign weighted $v_{1}^{*}$ for baryons (upper panel) and effective EoS (lower panel) in Au+Au collision at $\sqrt{s_{N N}}=11.5$ $\mathrm{GeV}$ from JAM simulations in which attractive orbits are imposed at different time intervals; the circles correspond to the simulation with attractive orbits for time $t<1 \mathrm{fm} / c$, the squares for $1<t<2 \mathrm{fm} / c$, and the triangles for $t>2 \mathrm{fm} / c$.

Effective EoS for each simulation are plotted together with the EoS of the standard JAM simulation in the lower panel of 
Fig. 9. The effect of the attractive orbits for $t<1 \mathrm{fm} / c$ is the slight reduction of pressure at high energy densities; on the other hand, attractive orbits for $t>2 \mathrm{fm} / c$ reduce the pressure at lower energy densities. Attractive orbits at $1<t<2 \mathrm{fm} / c$ strongly reduces the pressure at high energy densities which results in the collapse of proton directed flow. However, it does not necessarily imply that the equilibrium EoS at high energy density at high baryon density needs to be very soft for the negative $v_{1}$, since most of pressures at energy densities above $4 \mathrm{GeV} / \mathrm{fm}^{3}$ are extracted from preequilibrium stages of the reaction in the JAM simulation. Nonequilibrium effects need to be examined. Nevertheless, it suggests the need of a nonstandard dynamical effect which is related to the reduction of pressure at high baryon densities.

This analysis strongly suggests the importance of reaction dynamics at high baryon density. The time period of $1<t<2 \mathrm{fm} / \mathrm{c}$ at $\sqrt{s_{N N}}=11.5 \mathrm{GeV}$ relevant to the negative flow corresponds to the preequilibrium stage in the JAM hadronic transport approach, even though produced hadrons start to interact with each other. We do not have partonic interactions, unlike the PHSD model [23]. The effects of partonic interactions in the early stages of the reaction should be examined elsewhere in order to understand the dynamical effects on the directed flow.

\section{E. Elliptic flow}

In order to see systematics on the use of the attractive orbits in the scattering style, it is important to check other flow harmonics such as elliptic flow $v_{2}=\langle\cos (2 \phi)\rangle$. In Fig. 10. JAM cascade results are compared with the pseudorapidity dependence of $v_{2}$ for charged hadrons in midcentral (10-40\%) Au+Au collisions at $\sqrt{s_{N N}}=7.7,11.5,19.6$ and $27 \mathrm{GeV}$ [47]. It is seen that $v_{2}$ at midrapidity is not modified by the attractive orbits scattering style, but it underestimates the data about $20-30 \%$. Therefore, we do not see any softening effects on $v_{2}$ within our approach. Underestimation of $v_{2}$ by our approach suggests the need of partonic interactions in the early stages of the reactions. We have also studied other inclusive hadronic observables such as transverse momentum distributions and rapidity distributions and found that the effect of attractive orbits on them is very small.

It is also important to examine other observables such as the net-baryon number cumulants in the same energy range [48]. If the softening of the EoS comes from criticality around the critical point, divergence of cumulants appears as oscillating behavior as a result of smearing by the finite quark mass [52] or finite volume [53]. Thus it is an interesting question whether dynamical model calculations with the EoS softening can describe the observed nonmonotonic behavior of cumulant ratios [48]. Recently, it was shown that JAM with attractive orbits as well as nuclear mean-field effects does not describe the observed large enhancement of cumulant ratios [49].

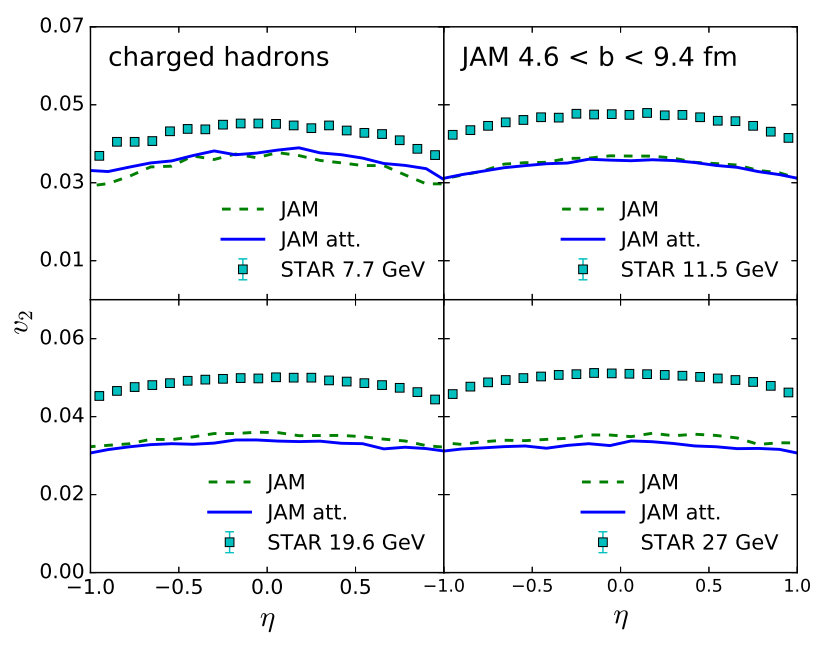

FIG. 10: Elliptic flows of charged hadrons in midcentral Au+Au collisions (10-40\%). The dotted lines correspond to the result from the standard JAM cascade model, while the solid lines are for the JAM cascade with attractive orbit results. Symbols show STAR data [47].

\section{F. EoS dependence of directed flow}

So far, we implement attractive orbits in JAM for all hadron-hadron $2 \rightarrow 2$ scatterings without any restrictions. As a result, our equation of state is soft for all energy densities as shown in Fig. 4. We now explore the EoS dependence of the directed flow. For this purpose, instead of imposing attractive scattering all the way, we select attractive orbits at each two-body scattering with the probability $p_{\text {attractive }}$ given by

$$
p_{\text {attractive }}=\max \left(0, \frac{P_{f}-P(e)}{P(e)}\right) \text {, }
$$

where $P(e)$ is a pressure as a function of energy density $e$ from a given EoS, and $P_{f}$ is the local pressure at the collision point computed from the energy-momentum tensor as in Sec. IIIB The QCD equation of state at high baryon densities is not well understood. As a first step, we use the EoS which does not depend on baryon density for simplicity, since our purpose here is to check the systematics of our approach that modifies the scattering style. We test the EoS with crossover from lattice QCD ( $s 95 p$-v1.1) taken from Ref. [50, 51], and the EoS with a first-order phase transition similar to that of EOS-Q with the modification of the slope in the QGP phase to $p=e / 3.5$ (instead of the massless ideal gas $\operatorname{EoS} p=e / 3$ ) so that pressure at high energy densities is consistent with the lattice EoS as shown in Fig. 11. We also show in Fig. 11 the results which are extracted from JAM simulations to ensure that our simple approach is consistent with a given EoS. It is seen that our simple approach works very well to modify the EoS of the system.

In Fig. 12 we plot the rapidity dependence of the directed flow of protons (upper panel) and pions (lower panel) in $\mathrm{Au}+\mathrm{Au}$ semicentral collision at $\sqrt{s_{N N}}=11.5 \mathrm{GeV}$ obtained from the EoS with crossover and first-order phase transition. While pion flow is not sensitive to the EoS, one sees that both 


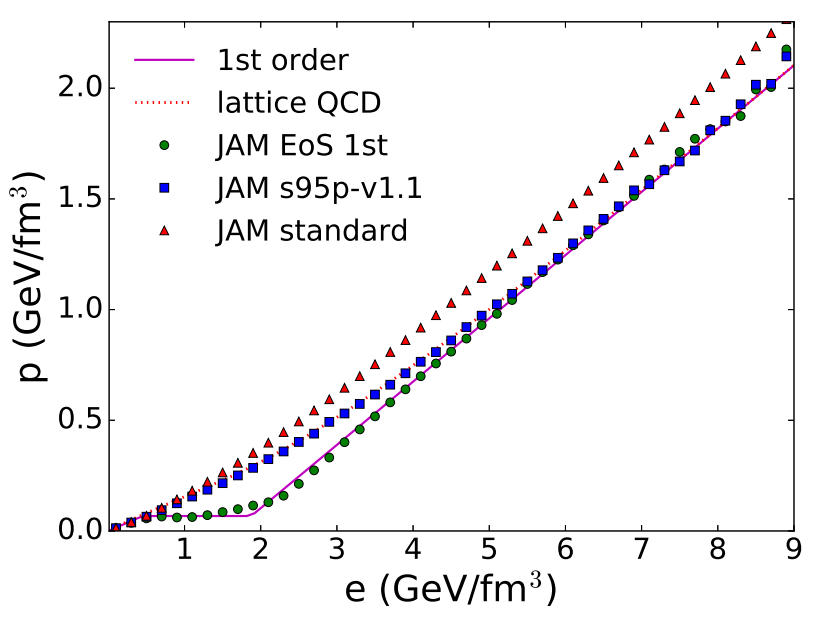

FIG. 11: Equation of state implemented into JAM simulations. The sold line represents the first-order phase transition EoS, and the dotted line represents the EoS from lattice QCD [50]. The circles and squares are results obtained by the JAM simulations, which are almost identical to the inputs. Effective EoS obtained from JAM standard simulation is also displayed by the triangles.

EoSs yield the suppression of proton directed flow compared to the standard cascade simulation, since both EoSs are softer than the effective EoS from the standard JAM cascade simulation as compared in Fig. 12, Proton flow at midrapidity $|y|<1.0$ is not sensitive to the EoS, but two EoS give different behavior for larger rapidities indicating that the softening point of the current EoS is responsible to the rapidity $|y|>1.0$ at $\sqrt{s_{N N}}=11.5 \mathrm{GeV}$, and explicit EoS dependence of the directed flow may be observable in the experiments. It remains for further work to establish the EoS dependence of the directed flow by utilizing a fully baryon density dependent EoS. The interactions used here do not employ any baryon density dependent interactions, $U(\rho)$, as one may want to use in relativistic mean-field models of high baryon density matter. It is also possible that $\Delta$ matter with slightly nonuniversal scalar attraction can easily cause a first-order phase transition without mentioning any high baryon density QCD. We will present a detailed systematic study of the EoS dependence of the directed flow elsewhere.

\section{SUMMARY}

In summary, we have investigated the effect of the softening of the EoS on the directed flow of protons and pions within a microscopic transport approach. The transport model JAM with standard stochastic two-body scattering style predicts the large positive slope of proton $v_{1}$ at collision energy below $\sqrt{s_{N N}}=19.6 \mathrm{GeV}$, and the negative slope of proton $v_{1}$ only at higher collision energy $\sqrt{s_{N N}} \geq 27 \mathrm{GeV}$, which disagree with the STAR data. However, softening effects of the EoS simulated by attractive orbit scatterings lead to a dramatic change in the dynamics, and yield significant reduction of proton $v_{1}$ which well describe the STAR data around the

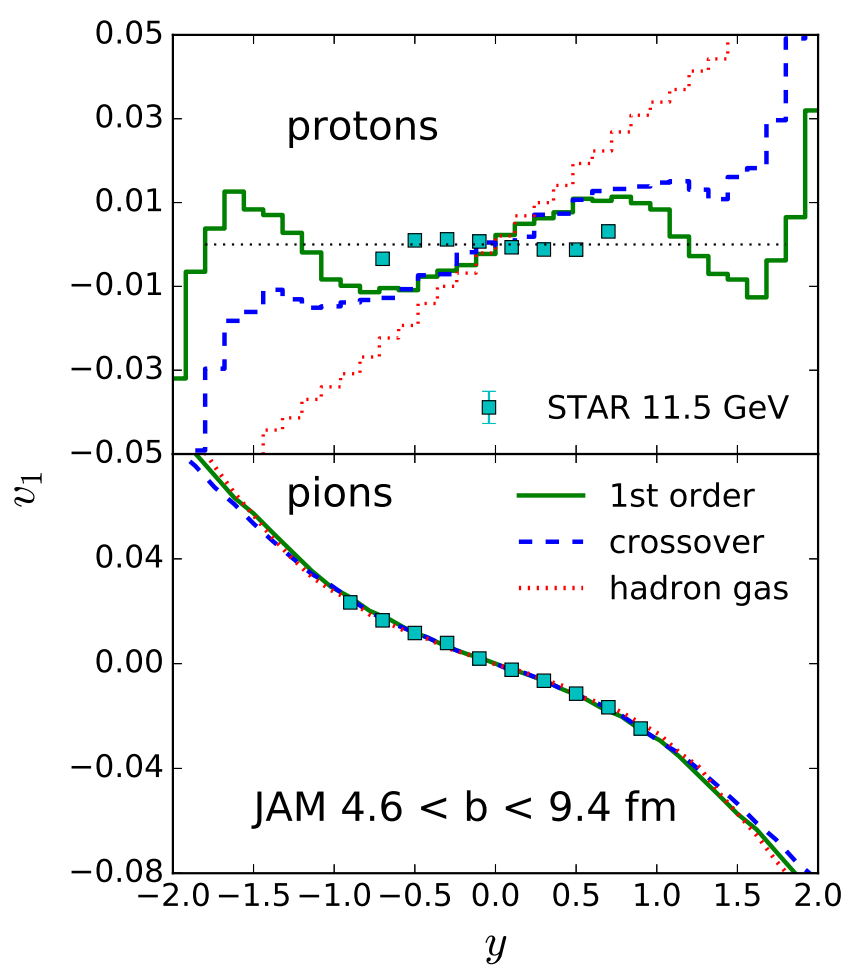

FIG. 12: Rapidity distribution of directed flow $v_{1}$ for protons and pions in semicentral $\mathrm{Au}+\mathrm{Au}$ collisions for $\sqrt{s_{N N}}=11.5 \mathrm{GeV}$ from JAM simulations with different EoS. The solid line presents the JAM result with the EoS with fist-order phase transition, and the dashed line presents the JAM result with the crossover EoS. The dotted line is for the standard JAM result.

minimum of $d v_{1} / d y$ at $10 \lesssim \sqrt{s_{N N}} \lesssim 20 \mathrm{GeV}$. The softening effects were not needed in the present approach at lower energies, $\sqrt{s_{N N}}=7.7 \mathrm{GeV}$. We found that attractive orbit scattering style does not modify elliptic flow at mid-rapidity. We show that this softening effect is needed only at early stages of the reaction where the system reaches the high baryon density state at midrapidity.

We also proposed a simple recipe to simulate a given EoS within a hadronic transport model, and compared two different EoS. We saw an EoS dependence of the proton directed flow in the forward rapidities. More detailed systematic studies are needed, using a fully baryon density dependent EoS, in order to draw a conclusion that the minimum of $d v_{1} / d y$ is a result of the softening of the EoS which may be caused by a first-order phase transition [11, 18, 37, 39].

A possible scenario to fully explain the beam energy dependence of the directed flow may be described as follows. We assume that there exists the softest point in the energy density range reachable at $\sqrt{s_{N N}}=11.5 \mathrm{GeV}$. Hadrons will feel an attractive force when they go across the surface of the soft region. This additional force can be simulated by introducing the attractive orbit scatterings among hadrons, as we have demonstrated in the present work, and negative $d v_{1} / d y$ emerges. One may need to introduce new degrees of freedom other than hadrons to understand the rehardening at higher energies. 
It seems obvious to infer a softening of the EoS from the experimentally observed collapse of net-proton flow when the c.m. energy is increased from 7 to $11 \mathrm{GeV}$. However, the statement of a discovery of the "softening" of the EoS from the net-proton $v_{1}$ data shows even more convincing evidence for the "phase transition" as we observe the rebound at higher energies; namely the STAR-observed second change of sign of the $v_{1}$ values of the net protons at $\sqrt{s_{N N}} \approx 40 \mathrm{GeV}$ back to positive $v_{1}$ at higher energies [1]. This shows that the soft region is overcome, and the directed flow picks up steam again, due to the rehardening of the EoS at considerably larger energy densities.

In the near future, a more detailed analysis of the softening effect should be addressed by employing a realistic EoS which is consistent with the lattice QCD result. Because of the nonequilibrium evolution, the pressure generation due to the two-body collision $\Delta P$ depends in the current study not only on the difference between the equilibrium EoSs, but also on the dynamical evolution of the system. Perhaps a more justified way of fixing the EoS would be to look at a fully equilibrated system in a box, and then determine the probabilities for attractive orbits as a function of the energy of the colliding particles, so that a given (equilibrium) EoS would be reproduced; then the microscopic dynamics would tell how the system looks in out-of-equilibrium situations.
It is expected that properties of the EoS at high baryon density may be probed sensitively by using the flow. Future experiments such as the BES II at RHIC [54], FAIR [55], NICA [56], and J-PARC [57] should clarify this point at lower collision energies $\sqrt{s_{N N}} \leq 10 \mathrm{GeV}$.

\section{Acknowledgement}

We would like to thank Adrian Dumitru for valuable comments. H.S. thanks $\mathrm{Nu} \mathrm{Xu}$, Zangbu Xu, Declan Keane, and Paul Sorensen for numerous useful discussions. Y.N. thanks the Frankfurt Institute of Advanced Studies where part of this work was done. This work was supported in part by the Grants-in-Aid for Scientific Research from JSPS (No. 15K05079, No. $15 \mathrm{H} 03663$ and No. 15K05098), the Grants-in-Aid for Scientific Research on Innovative Areas from MEXT (No. 24105001 and No. 24105008), and by the Yukawa International Program for Quark-Hadron Sciences. H.N. has received funding from the European Union's Horizon 2020 Research and Innovation Programme under Marie Sklodowska-Curie Grant Agreement No. 655285 and from the Helmholtz International Center for FAIR within the framework of the LOEWE program launched by the State of Hesse.
[1] L. Adamczyk et al. [STAR Collaboration], Phys. Rev. Lett. 112, no. 16, 162301 (2014).

[2] I. Arsene et al. [BRAHMS Collaboration], Nucl. Phys. A 757, 1 (2005); B. B. Back et al., Nucl. Phys. A 757, 28 (2005); J. Adams et al. [STAR Collaboration], Nucl. Phys. A 757, 102 (2005); K. Adcox et al. [PHENIX Collaboration], Nucl. Phys. A 757, 184 (2005).

[3] Y. Aoki, G. Endrodi, Z. Fodor, S. D. Katz and K. K. Szabo, Nature 443, 675 (2006).

[4] D. H. Rischke, Prog. Part. Nucl. Phys. 52, 197 (2004); M. A. Stephanov, Prog. Theor. Phys. Suppl. 153, 139 (2004) [Int. J. Mod. Phys. A 20, 4387 (2005)]; K. Fukushima and T. Hatsuda, Rept. Prog. Phys. 74, 014001 (2011); K. Fukushima and C. Sasaki, Prog. Part. Nucl. Phys. 72, 99 (2013).

[5] C. M. Hung and E. V. Shuryak, Phys. Rev. Lett. 75, 4003 (1995).

[6] D. H. Rischke, Y. Pursun, J. A. Maruhn, H. Stoecker and W. Greiner, Heavy Ion Phys. 1, 309 (1995).

[7] M. M. Aggarwal et al. [STAR Collaboration], arXiv:1007.2613 [nucl-ex].

[8] L. Kumar, Mod. Phys. Lett. A 28, 1330033 (2013).

[9] J. Hofmann, H. Stoecker, U. W. Heinz, W. Scheid and W. Greiner, Phys. Rev. Lett. 36, 88 (1976).

[10] H. Stoecker and W. Greiner, Phys. Rept. 137, 277 (1986).

[11] H. Stoecker, Nucl. Phys. A 750, 121 (2005).

[12] H. G. Baumgardt, J. U. Schott, Y. Sakamoto, E. Schopper, H. Stoecker, J. Hofmann, W. Scheid and W. Greiner, Z. Phys. A 273, 359 (1975).

[13] W. Scheid, H. Müller and W. Greiner, Phys. Rev. Lett. 32, 741 (1974); J. Kapusta and D. Strottman, Phys. Lett. B 106, 33 (1981); H. Stoecker et al., Phys. Rev. C 25, 1873 (1982).

[14] H. A. Gustafsson et al., Phys. Rev. Lett. 52, 1590 (1984).
[15] Y. B. Ivanov, E. G. Nikonov, W. Noerenberg, A. A. Shanenko and V. D. Toneev, Heavy Ion Phys. 15, 117 (2002); V. D. Toneev, E. G. Nikonov, B. Friman, W. Norenberg and K. Redlich, Eur. Phys. J. C 32, 399 (2003).

[16] L. P. Csernai and D. Rohrich, Phys. Lett. B 458, 454 (1999).

[17] L. P. Csernai, A. Anderlik, C. Anderlik, V. K. Magas, E. Molnar, A. Nyiri, D. Rohrich and K. Tamosiunas, Acta Phys. Hung. A 22, 181 (2005).

[18] J. Brachmann, S. Soff, A. Dumitru, H. Stoecker, J. A. Maruhn, W. Greiner, L. V. Bravina and D. H. Rischke, Phys. Rev. C 61, 024909 (2000).

[19] J. Brachmann, A. Dumitru, H. Stoecker and W. Greiner, Eur. Phys. J. A 8, 549 (2000).

[20] P. Shanmuganathan [STAR Collaboration], arXiv:1512.09009 [nucl-ex].

[21] R. J. M. Snellings, H. Sorge, S. A. Voloshin, F. Q. Wang and N. Xu, Phys. Rev. Lett. 84, 2803 (2000).

[22] M. Bleicher and H. Stoecker, Phys. Lett. B 526, 309 (2002).

[23] V. P. Konchakovski, W. Cassing, Y. B. Ivanov and V. D. Toneev, Phys. Rev. C 90, no. 1, 014903 (2014).

[24] H. Petersen, Q. Li, X. Zhu and M. Bleicher, Phys. Rev. C 74, 064908 (2006).

[25] J. Steinheimer, J. Auvinen, H. Petersen, M. Bleicher and H. Stoecker, Phys. Rev. C 89, no. 5, 054913 (2014).

[26] H. Liu et al. [E895 Collaboration], Phys. Rev. Lett. 84, 5488 (2000).

[27] H. Appelshauser et al. [NA49 Collaboration], Phys. Rev. Lett. 80, 4136 (1998).

[28] C. Alt et al. [NA49 Collaboration], Phys. Rev. C 68, 034903 (2003).

[29] M. Isse, A. Ohnishi, N. Otuka, P. K. Sahu and Y. Nara, Phys. Rev. C 72, 064908 (2005). 
[30] Y. B. Ivanov and A. A. Soldatov, Phys. Rev. C 91, no. 2, 024915 (2015).

[31] B. A. Li and C. M. Ko, Phys. Rev. C 58, 1382(R) (1998).

[32] Y. Nara, N. Otuka, A. Ohnishi, K. Niita and S. Chiba, Phys. Rev. C 61, 024901 (2000).

[33] H. Sorge, Phys. Rev. C 52, 3291 (1995).

[34] S. A. Bass et al., Prog. Part. Nucl. Phys. 41, 255 (1998).

[35] M. Bleicher et al., J. Phys. G 25, 1859 (1999).

[36] E. C. Halbert, Phys. Rev. C 23, 295 (1981).

[37] M. Gyulassy, K. A. Frankel and H. Stoecker, Phys. Lett. B 110, 185 (1982).

[38] D. E. Kahana, D. Keane, Y. Pang, T. Schlagel and S. Wang, Phys. Rev. Lett. 74, 4404 (1995); D. E. Kahana, Y. Pang and E. V. Shuryak, Phys. Rev. C 56, 481 (1997).

[39] H. Sorge, Phys. Rev. Lett. 82, 2048 (1999).

[40] P. Danielewicz and S. Pratt, Phys. Rev. C 53, 249 (1996).

[41] S. A. Bass, C. Hartnack, H. Stoecker and W. Greiner, Phys. Rev. C 51, 3343 (1995) doi:10.1103/PhysRevC.51.3343 [nucl-th/9501002].

[42] Y. Nara and A. Ohnishi, arXiv:1512.06299 [nucl-th].

[43] K. Werner, I. Karpenko, T. Pierog, M. Bleicher and K. Mikhailov, Phys. Rev. C 82, 044904 (2010).

[44] P. F. Kolb, J. Sollfrank and U. W. Heinz, Phys. Lett. B 459, 667 (1999); P. F. Kolb, J. Sollfrank and U. W. Heinz, Phys. Rev. C
62, 054909 (2000); H. Song and U. W. Heinz, Phys. Rev. C 77, 064901 (2008).

[45] L. V. Bravina et al., Phys. Rev. C 60, 024904 (1999); Phys. Rev. C 78, 014907 (2008).

[46] H. Sorge, Phys. Lett. B 402, 251 (1997)

[47] L. Adamczyk et al. [STAR Collaboration], Phys. Rev. C 86, 054908 (2012).

[48] L. Adamczyk et al. [STAR Collaboration], Phys. Rev. Lett. 112, 032302 (2014).

[49] S. He, X. Luo, Y. Nara, S. Esumi and N. Xu, arXiv:1607.06376 [nucl-ex].

[50] P. Huovinen and P. Petreczky, Nucl. Phys. A 837, 26 (2010). https://wiki.bnl.gov/hhic/index.php/Lattice_calcula

[51] T. Hirano, P. Huovinen, K. Murase and Y. Nara, Prog. Part. Nucl. Phys. 70, 108 (2013).

[52] B. Friman, F. Karsch, K. Redlich and V. Skokov, Eur. Phys. J. C 71, 1694 (2011).

[53] T. Ichihara, K. Morita and A. Ohnishi, Prog. Theor. Exp. Phys. 2015, 113D01 (2015).

[54] G. Odyniec, EPJ Web Conf. 95, 03027 (2015).

[55] http: //www.fair-center.eu//

[56] http://nica.jinr.ru/

[57] H. Sako et al., Nucl. Phys. A 931, 1158 (2014). 